\title{
Understanding Aluminium Location and Non-framework Ions Effects on Alkane Adsorption in Aluminosilicates: a Molecular Simulation Study
}

Bei Liu, Elena García-Pérez, David Dubbeldam, Berend Smit, and Sofía Calero

\section{Supporting Information}

In this work, we firstly categorized the commonly used zeolites into the two groups by computing the Henry coefficients of linear alkanes in FAU, LTA, MEL, MFI, LTL, FER, MOR, and TON zeolitic structures, and the results are shown in Figure 1. In the simulations the $\mathrm{Si} / \mathrm{Al}$ ratio is kept fixed for each zeolite by varying the aluminium distribution for different structures (the details about the exact location of the $\mathrm{Al}$ atoms in the different structures are shown in Tables 1-5) and using $\mathrm{Na}^{+}$and $\mathrm{H}^{+}$ as non-framework ions. The pure silica structures were constructed by using the crystallographic coordinates reported ${ }^{50}$ and the structures with aluminium were obtained by randomly replacing silicon by aluminium atoms, satisfying the Löwenstein rule. 
a) FAU $-52 \mathrm{Na}^{+} / \mathrm{uc}$

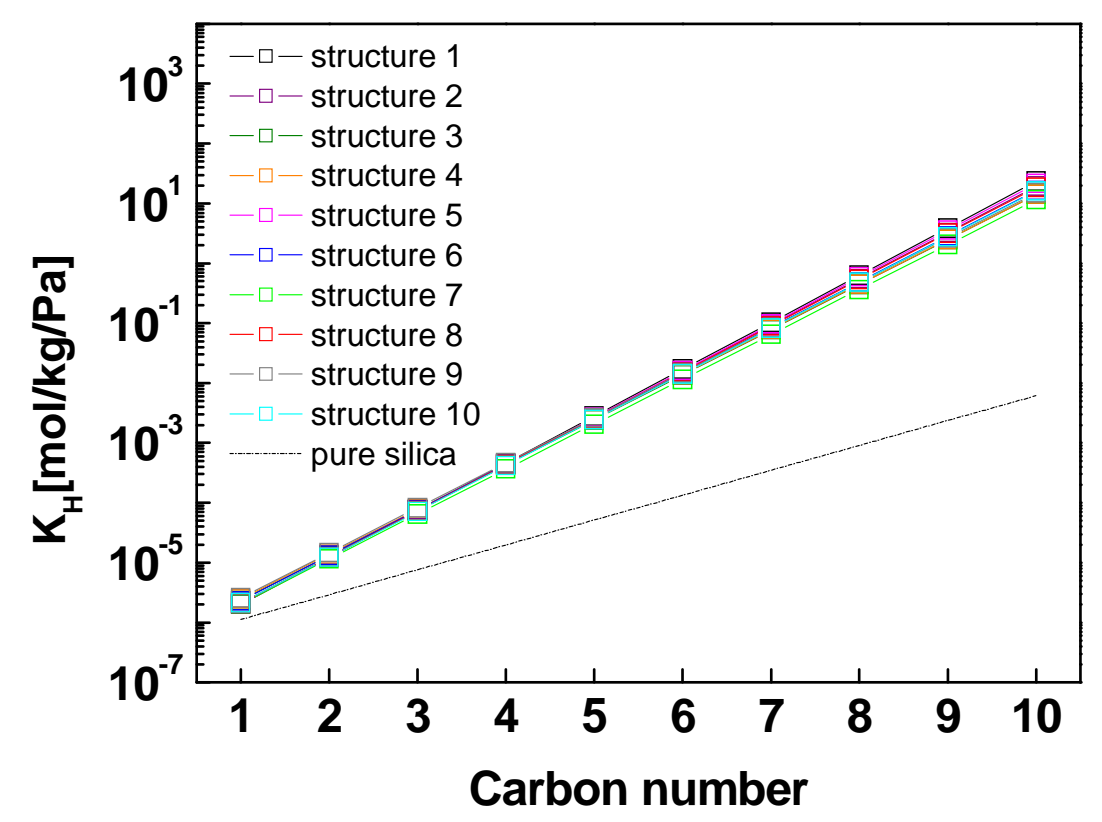

b) LTA - $48 \mathrm{Na}^{+} / \mathrm{uc}$

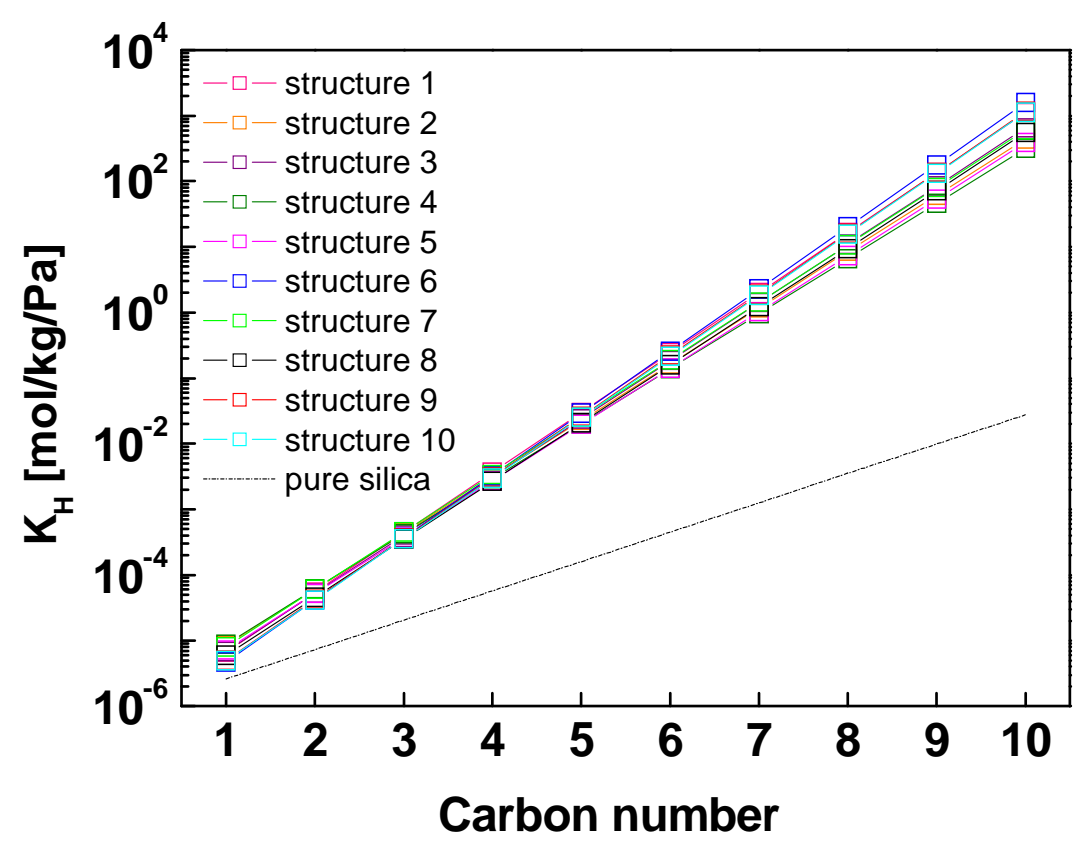


c) MEL $-4 \mathrm{Na}^{+} / \mathrm{uc}$

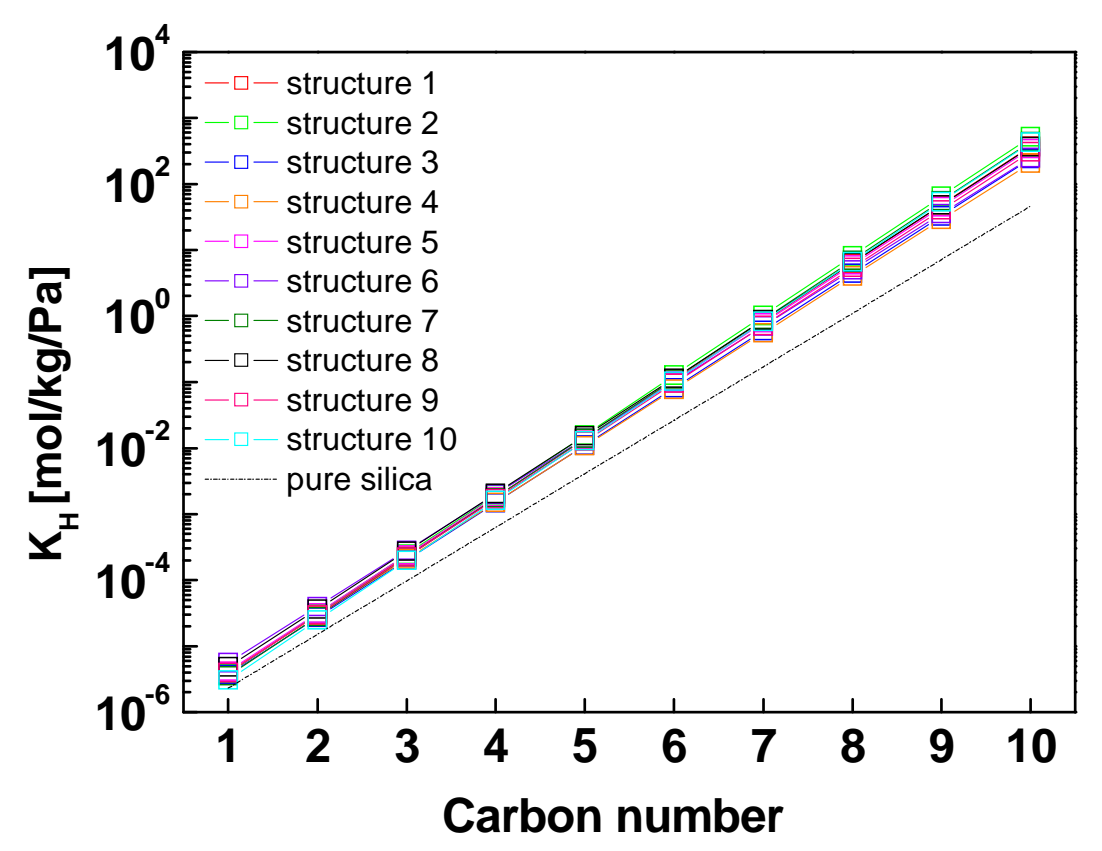

d) $\mathrm{MFI}-4 \mathrm{Na}^{+} / \mathrm{uc}$

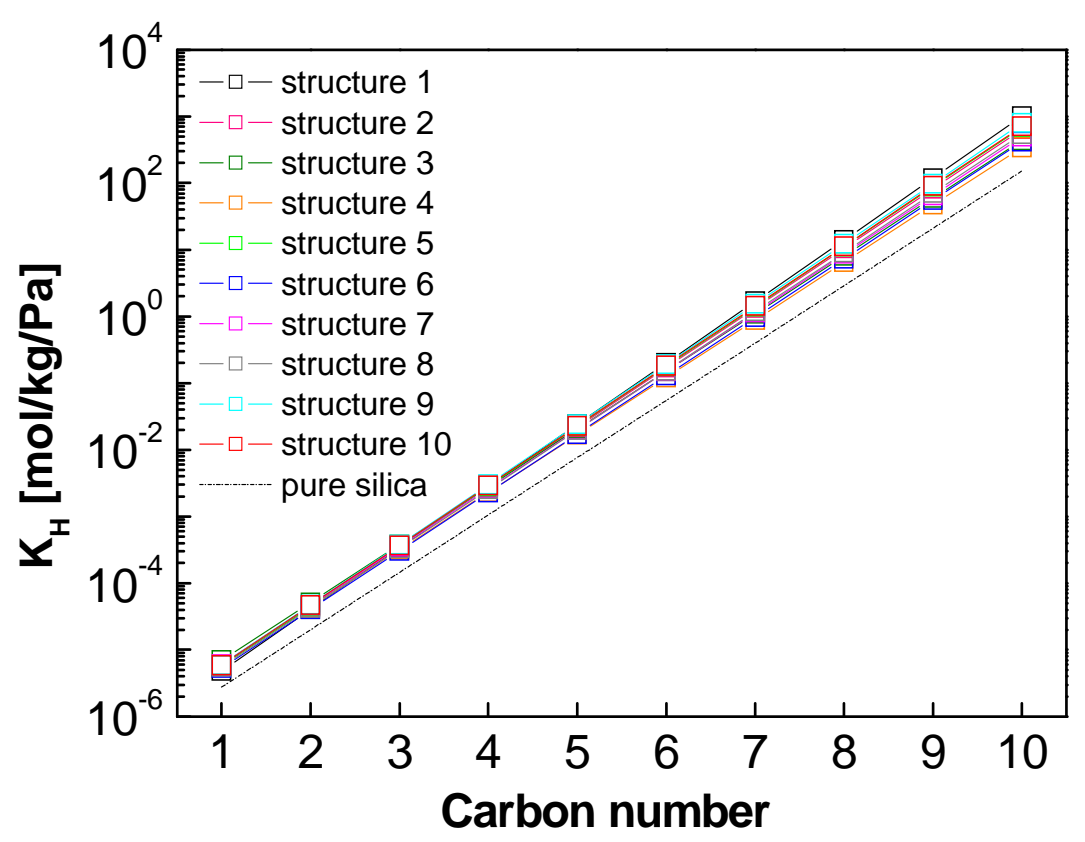


e) LTL - $6 \mathrm{Na}^{+} / \mathrm{uc}$

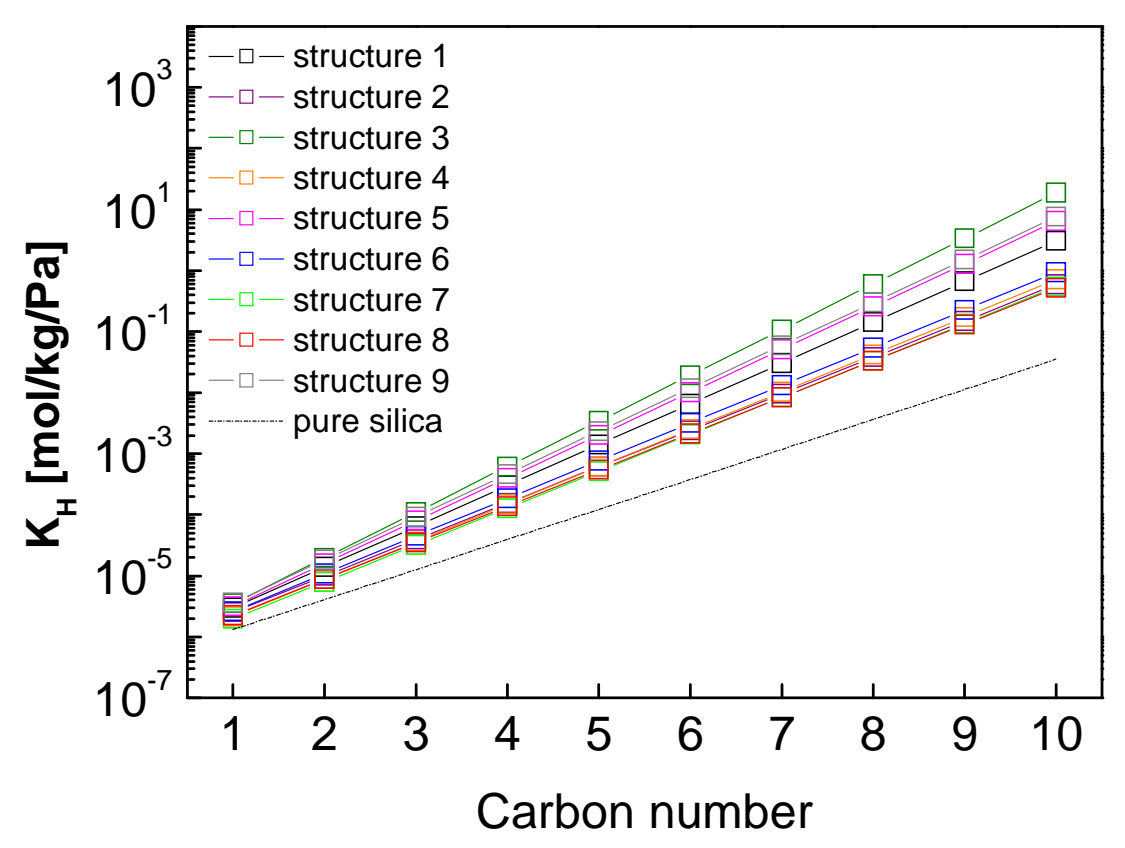

f) FER $-4 \mathrm{Na}^{+} / \mathrm{uc}$

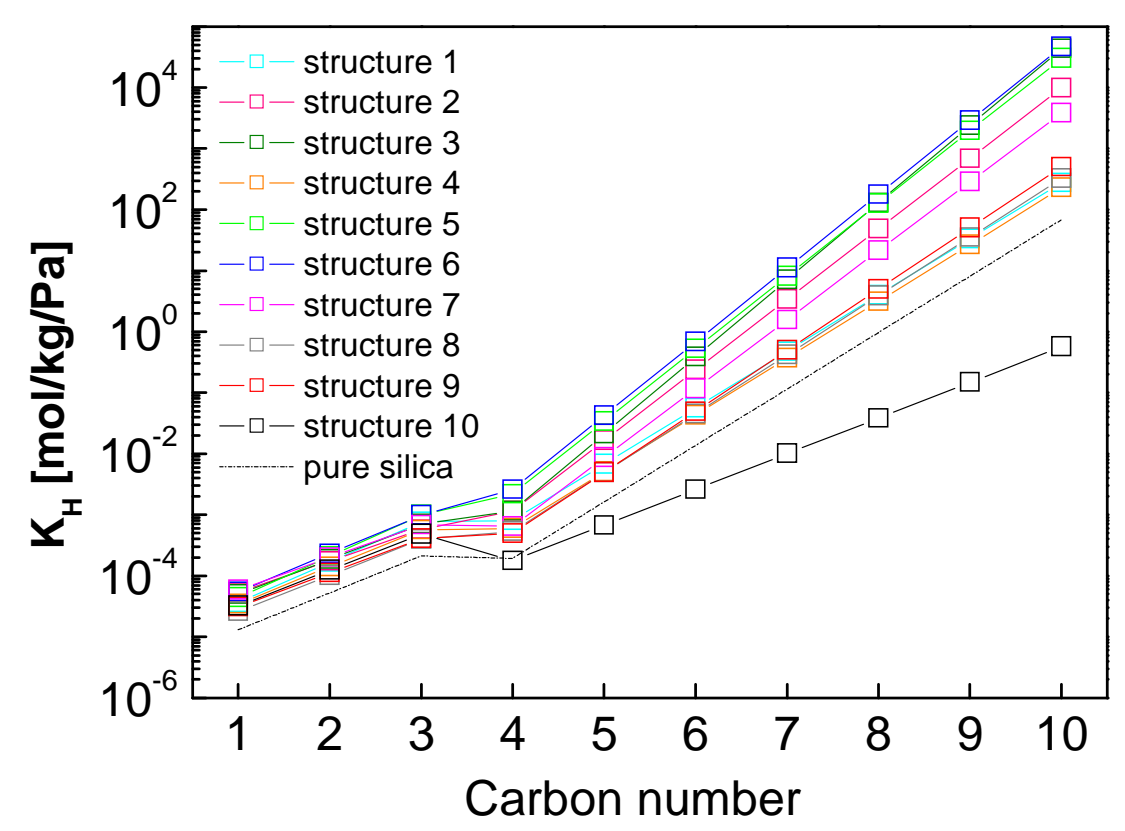


g) $M O R-4 \mathrm{Na}^{+} / \mathrm{uc}$

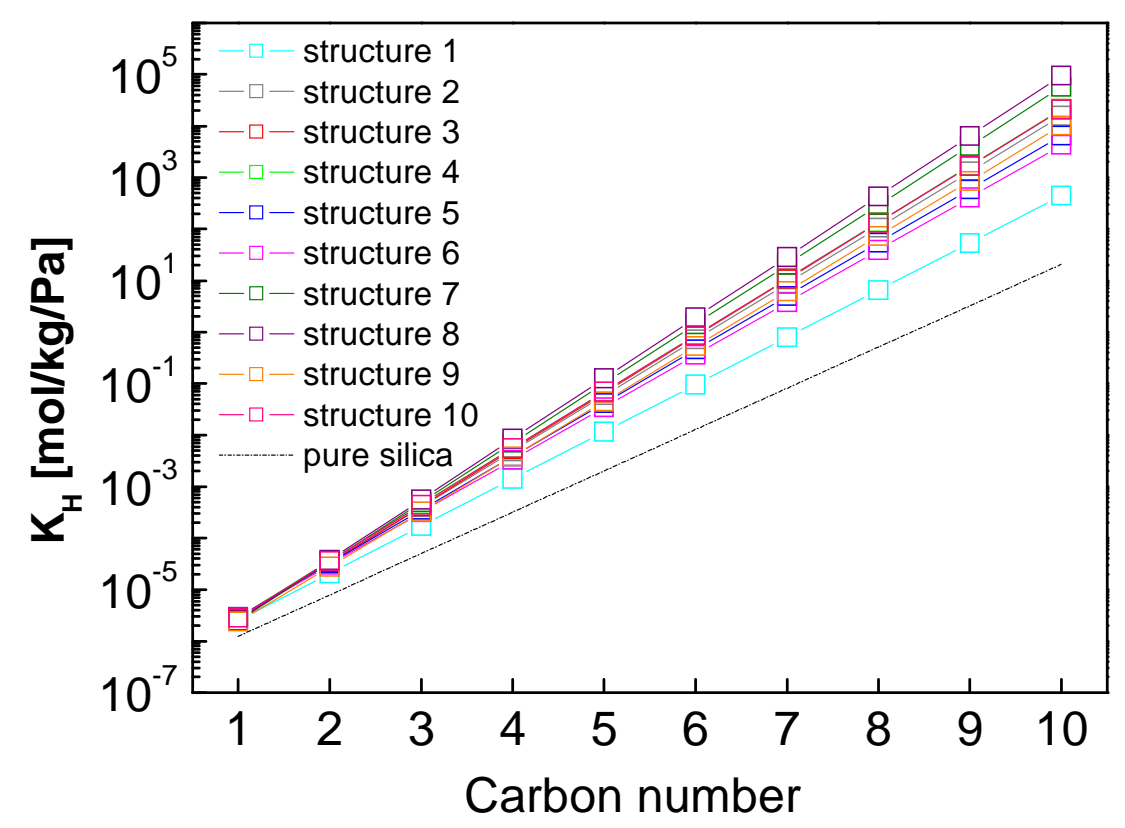

h) $\mathrm{TON}-1 \mathrm{H}^{+} / \mathrm{uc}$

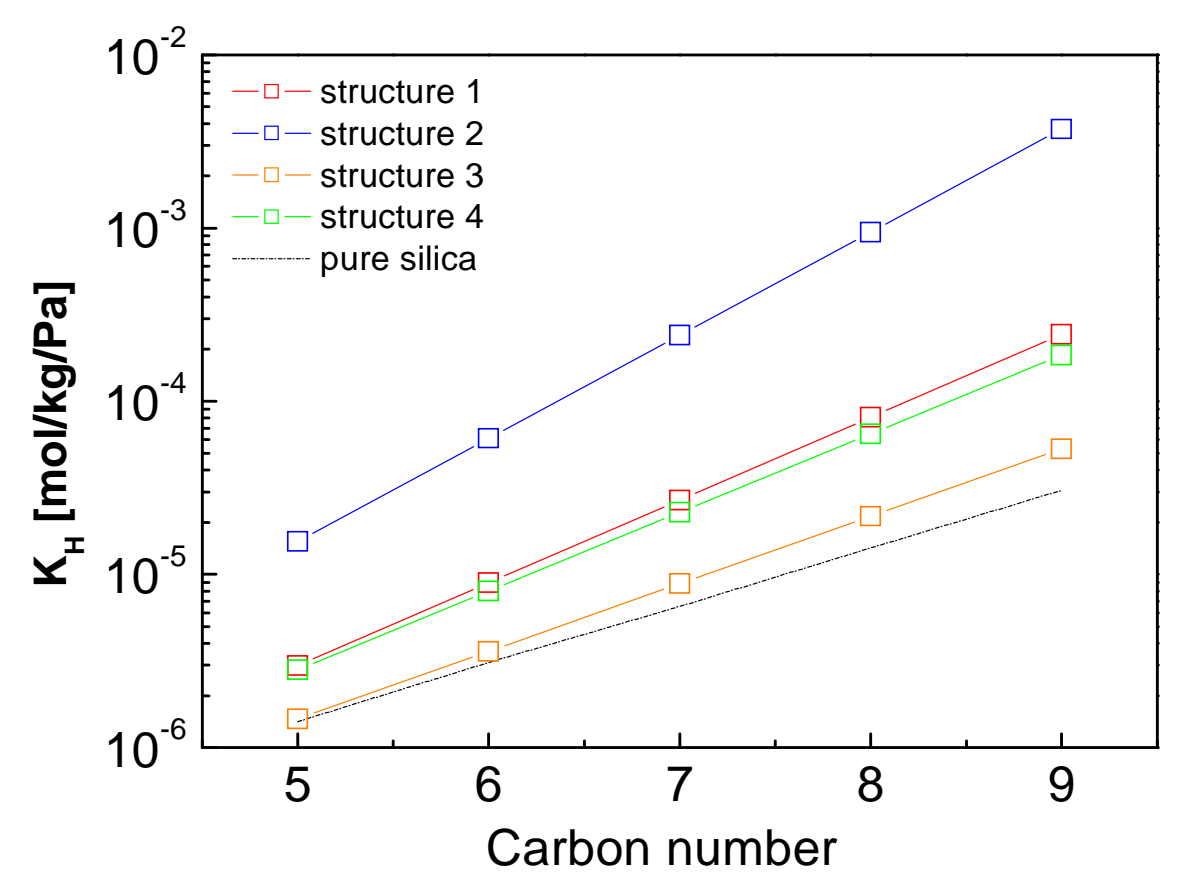

Fig.1 Henry coefficients of linear alkanes in a) FAU, b) LTA, c) MEL, d) MFI, e) LTL, f) FER, g) MOR zeolites at $350 \mathrm{~K}$ and h) TON zeolite at $548 \mathrm{~K}$. 
TABLE 1: Structural Information for FAU zeolite considered in this work

\begin{tabular}{|c|c|}
\hline & Al location sites \\
\hline Structure 1 & $\begin{array}{l}\text { Si2, Si26, Si38, Si44, Si62, Si68, Si86, Si92, Si98, Si110, Si134, Si140, Si158, Si164, Si176, } \\
\text { Si182, Si188, Si194, Si224, Si230, Si236, Si242, Si248, Si272, Si284, Si290, Si314, Si332, Si344, } \\
\text { Si350, Si356, Si368, Si380, Si392, Si404, Si410, Si416, Si422, Si428, Si434, Si440, Si452, Si458, } \\
\text { Si470, Si476, Si500, Si506, Si512, Si536, Si542, Si554, and Si560. }\end{array}$ \\
\hline Structure 2 & $\begin{array}{l}\text { Si2, Si8, Si14, Si20, Si26, Si44, Si50, Si68, Si74, Si80, Si92, Si104, Si110, Si116, Si128, Si134, } \\
\text { Si146, Si152, Si164, Si170, Si188, Si218, Si230, Si236, Si242, Si248, Si254, Si278, Si284, Si308, } \\
\text { Si314, Si332, Si338, Si344, Si350, Si356, Si374, Si386, Si398, Si404, Si422, Si434, Si446, Si452, } \\
\text { Si494, Si500, Si506, Si512, Si530, Si542, Si560, and Si572. }\end{array}$ \\
\hline Structure 3 & $\begin{array}{l}\text { Si14, Si38, Si50, Si56, Si62, Si68, Si80, Si92, Si116, Si140, Si158, Si164, Si182, Si188, Si194, } \\
\text { Si200, Si230, Si236, Si242, Si248, Si260, Si284, Si290, Si302, Si314, Si326, Si332, Si344, Si356, } \\
\text { Si362, Si374, Si392, Si404, Si410, Si428, Si434, Si440, Si452, Si458, Si464, Si470, Si476, Si482, } \\
\text { Si488, Si494, Si500, Si512, Si518, Si530, Si536, Si542, and Si572. }\end{array}$ \\
\hline Structure 4 & $\begin{array}{l}\text { Si2, Si8, Si20, Si26, Si44, Si56, Si80, Si104, Si110, Si122, Si128, Si134, Si140, Si146, Si152, } \\
\text { Si176, Si182, Si188, Si194, Si206, Si218, Si224, Si230, Si254, Si260, Si272, Si284, Si296, Si302, } \\
\text { Si314, Si332, Si344, Si368, Si380, Si386, Si398, Si404, Si410, Si416, Si440, Si446, Si452, Si458, } \\
\text { Si470, Si476, Si488, Si494, Si530, Si542, Si548, Si554, and Si566. }\end{array}$ \\
\hline Structure 5 & $\begin{array}{l}\text { Si2, Si8, Si14, Si26, Si32, Si38, Si50, Si56, Si74, Si92, Si98, Si104, Si128, Si140, Si152, Si158, } \\
\text { Si164, Si170, Si176, Si182, Si188, Si200, Si206, Si218, Si224, Si236, Si254, Si260, Si284, Si290, } \\
\text { Si314, Si320, Si338, Si344, Si368, Si374, Si392, Si428, Si434, Si440, Si452, Si464, Si476, Si488, } \\
\text { Si494, Si500, Si506, Si518, Si530, Si548, Si554, and Si566. }\end{array}$ \\
\hline Structure 6 & $\begin{array}{l}\text { Si56, Si62, Si74, Si86, Si98, Si104, Si122, Si134, Si140, Si152, Si158, Si182, Si188, Si194, } \\
\text { Si200, Si206, Si218, Si230, Si236, Si242, Si254, Si278, Si284, Si290, Si296, Si308, Si320, Si326, } \\
\text { Si332, Si344, Si350, Si356, Si362, Si368, Si380, Si392, Si404, Si410, Si416, Si428, Si440, Si452, } \\
\text { Si458, Si470, Si494, Si500, Si506, Si518, Si524, Si536. Si554, and Si566. }\end{array}$ \\
\hline Structure 7 & $\begin{array}{l}\text { Si14, Si20, Si50, Si56, Si68, Si86, Si104, Si110, Si116, Si122, Si128, Si134, Si152, Si158, Si164, } \\
\text { Si170, Si176, Si188, Si200, Si206, Si218, Si224, Si230, Si236, Si242, Si254, Si260, Si266, Si272, } \\
\text { Si302, Si308, Si314, Si326, Si332, Si344, Si356, Si362, Si368, Si404, Si422, Si434, Si440, Si458, } \\
\text { Si470. Si476, Si482, Si530, Si536, Si542, Si560, and Si572. }\end{array}$ \\
\hline Structure 8 & $\begin{array}{l}\text { Si44, Si50, Si56, Si62, Si74, Si86, Si104, Si110, Si116, Si128, Si140, Si146, Si152, Si170, Si176, } \\
\text { Si182, Si194, Si206, Si224, Si242, Si248, Si260, Si266, Si272, Si290, Si296, Si302, Si308, Si314, } \\
\text { Si320, Si332,Si338, Si344, Si350, Si362, Si368, Si374, Si380, Si398, Si410, Si416, Si428, Si434, } \\
\text { Si440, Si452, Si458, Si464, Si476, Si494, Si518, Si530, and Si542. }\end{array}$ \\
\hline Structure 9 & $\begin{array}{l}\text { Si8, Si32, Si38, Si44, Si50, Si56, Si62, Si68, Si80, Si86, Si98, Si110, Si128, Si134, Si140, Si164, } \\
\text { Si176, Si188, Si194, Si206, Si218, Si224, Si236, Si248, Si260, Si266, Si272, Si296, Si302, Si314, } \\
\text { Si326, Si344, Si368, Si380, Si386, Si392, Si416, Si440, Si446, Si458, Si464, Si470, Si476, Si494, } \\
\text { Si500, Si518, Si536, Si548, Si554, Si560, Si566, and Si572. }\end{array}$ \\
\hline Structure 10 & $\begin{array}{l}\text { Si14, Si56, Si62, Si74, Si80, Si86, Si98, Si104, Si122, Si134, Si140, Si152, Si158, Si164, Si182, } \\
\text { Si188, Si194, Si200, Si206, Si218, Si230, Si236, Si242, Si254, Si266, Si272, Si278, Si284, Si290, } \\
\text { Si296, Si308, Si320, Si326, Si332, Si338, Si344, Si350, Si356, Si362, Si368, Si380, Si392, Si404, } \\
\text { Si410, Si416, Si428, Si440, Si452, Si458, Si494, Si500, and Si506. }\end{array}$ \\
\hline
\end{tabular}


TABLE 2: Structural Information for LTA zeolite considered in this work

\begin{tabular}{|c|c|}
\hline & Al location sites \\
\hline re 1 & $\begin{array}{l}\text { Si17, Si27, Si47, Si52, Si57, Si72, Si77, Si87, Si102, Si112, Si171, Si181, Si191, Si196, Si211, } \\
\text { Si216, Si231, Si236, Si246, Si251, Si261, Si295, Si300, Si305, Si320, Si340, Si350, Si355, Si365, } \\
\text { Si375, Si380, Si385, Si400, Si439, Si454, Si469, Si474, Si479, Si484, Si499, Si504, Si519, Si524, } \\
\text { Si529, Si534, Si539, Si544, and Si549. }\end{array}$ \\
\hline 2 & $\begin{array}{l}\text { Si12, Si17, Si32, Si47, Si57, Si67, Si72, Si82, Si87, Si92, Si107, Si117, Si151, Si156, Si166, } \\
\text { Si171, Si181, Si186, Si196, Si201, Si211, Si221, Si226, Si231, Si241, Si246, Si251, Si261, Si295, } \\
\text { Si300, Si315, Si320, Si330, Si340, Si345, Si355, Si365, Si380, Si390, Si400, Si439, Si444, Si454, } \\
\text { Si474, Si494, Si514, Si524, and Si534. }\end{array}$ \\
\hline Struc & $\begin{array}{l}\text { Si2, Si22, Si32, Si42, Si52, Si62, Si72, Si82, Si92, Si102, Si112, Si146, Si156, Si161, Si171, } \\
\text { Si181, Si191, Si196, Si206, Si211, Si216, Si226, Si231, Si241, Si251, Si261, Si295, Si305, Si310, } \\
\text { Si315, Si325, Si335, Si350, Si360, Si370, Si375, Si385, Si395, Si405, Si439, Si444, Si454, Si489, } \\
\text { Si504, Si519, Si529, Si539, and Si544. }\end{array}$ \\
\hline Stru & $\begin{array}{l}\text { Si7, Si12, Si22, Si32, Si47, Si57, Si67, Si72, Si77, Si82, Si87, Si92, Si107, Si117, Si156, Si166, } \\
\text { Si176, Si181, Si191, Si196, Si201, Si211, Si221, Si231, Si236, Si246, Si251, Si261, Si300, Si310, } \\
\text { Si320, Si335, Si350, Si360, Si370, Si400, Si439, Si454, Si474, Si504, Si514, Si519, Si524, Si529, } \\
\text { Si534, Si539, Si544, and Si549. }\end{array}$ \\
\hline 5 & $\begin{array}{l}\text { Si12, Si67, Si77, Si87, Si117, Si156, Si166, Si206, Si216, Si221, Si241, Si290, Si295, Si300, } \\
\text { Si310, Si320, Si330, Si340, Si345, Si350, Si355, Si360, Si365, Si370, Si375, Si380, Si385, Si390, } \\
\text { Si395, Si400, Si405, Si434, Si439, Si444, Si449, Si454, Si459, Si464, Si469, Si494, Si504, Si514, } \\
\text { Si519, Si524, Si534, Si539, Si544, and Si549. }\end{array}$ \\
\hline Stru & $\begin{array}{l}\text { Si12, Si22, Si27, Si32, Si37, Si42, Si47, Si52, Si67, Si77, Si87, Si92, Si102, Si112, Si151, Si161, } \\
\text { Si166, Si181, Si191, Si196, Si201, Si206, Si226, Si231, Si290, Si300, Si315, Si325, Si335, Si345, } \\
\text { Si360, Si370, Si380, Si390, Si400, Si439, Si444, Si454, Si469, Si479, Si494, Si504, Si509, Si514, } \\
\text { Si534, Si539, Si544, and Si549. }\end{array}$ \\
\hline Stru & $\begin{array}{l}\text { Si2, Si7, Si12, Si17, Si27, Si37, Si47, Si52, Si57, Si67, Si72, Si82, Si87, Si97, Si107, Si117, } \\
\text { Si151, Si156, Si166, Si176, Si186, Si196, Si206, Si256, Si295, Si305, Si325, Si345, Si355, Si360, } \\
\text { Si370, Si380, Si385, Si400, Si439, Si444, Si454, Si464, Si469, Si479, Si489, Si499, Si504, Si514, } \\
\text { Si519, Si529, Si539, and Si549. }\end{array}$ \\
\hline Stru & $\begin{array}{l}\text { Si2, Si7, Si17, Si27, Si32, Si42, Si47, Si57, Si67, Si77, Si97, Si102, Si146, Si151, Si156, Si161, } \\
\text { Si166, Si181, Si196, Si201, Si206, Si211, Si221, Si231, Si241, Si246, Si251, Si261, Si300, Si305, } \\
\text { Si310, Si325, Si330, Si340, Si345, Si360, Si365, Si380, Si385, Si400, Si439, Si444, Si459, Si464, } \\
\text { Si484, Si494, Si509, and Si519. }\end{array}$ \\
\hline Struc & $\begin{array}{l}\text { Si7, Si17, Si27, Si42, Si72, Si87, Si107, Si151, Si156, Si166, Si176, Si181, Si191, Si196, Si206, } \\
\text { Si216, Si221, Si236, Si241, Si251, Si256, Si295, Si300, Si305, Si325, Si330, Si340, Si345, Si360, } \\
\text { Si370, Si380, Si395, Si405, Si439, Si444, Si449, Si454, Si459, Si464, Si474, Si479, Si489, Si499, } \\
\text { Si504, Si509, Si524, Si529, and Si539. }\end{array}$ \\
\hline Structure 10 & $\begin{array}{l}\text { Si2, Si12, Si17, Si27, Si32, Si47, Si72, Si77, Si82, Si87, Si92, Si97, Si102, Si107, Si112, Si117, } \\
\text { Si161, Si166, Si176, Si181, Si196, Si201, Si206, Si216, Si221, Si226, Si236, Si241, Si246, Si256, } \\
\text { Si261, Si290, Si295, Si305, Si310, Si315, Si330, Si345, Si355, Si395, Si400, Si454, Si479, Si484, } \\
\text { Si494, Si504, Si529, and Si534. }\end{array}$ \\
\hline
\end{tabular}


TABLE 3: Structural Information for MEL and MFI zeolites considered in this work

\begin{tabular}{|c|c|c|}
\hline \multirow{2}{*}{} & \multicolumn{2}{|c|}{ Al location sites } \\
\cline { 2 - 3 } & MEL & MFI \\
\hline Structure 1 & Si7, Si50, Si67, and Si125. & Si10, Si124, Si162, and Si264. \\
\hline Structure 2 & Si27, Si72, Si90, and Si178. & Si3, Si11, Si225, and Si263. \\
\hline Structure 3 & Si2, Si73, Si193, and Si278. & Si39, Si164, Si197, and Si260. \\
\hline Structure 4 & Si1, Si68, Si137, and Si153. & Si39, Si163, Si197, and Si266. \\
\hline Structure 5 & Si45, Si71, Si72, and Si125. & Si1, Si195, Si230, and Si265. \\
\hline Structure 6 & Si3, Si67, Si92, and Si137. & Si42, Si48, Si88, and Si259. \\
\hline Structure 7 & Si3, Si29, Si137, and Si153. & Si10, Si154, Si197, and Si266. \\
\hline Structure 8 & Si2, Si27, Si69, and Si122. & Si10, Si163, Si196, and Si265. \\
\hline Structure 9 & Si5, Si29, Si73, and Si125. & Si115, Si158, Si192, and Si221. \\
\hline Structure 10 & Si7, Si50, Si73, and Si92. & Si10, Si41, Si50, and Si83. \\
\hline
\end{tabular}

TABLE 4: Structural Information for LTL, FER, and TON zeolites considered in this work

\begin{tabular}{|c|c|c|c|}
\hline \multirow{2}{*}{} & \multicolumn{2}{|c|}{ Al location sites } & TON \\
\cline { 2 - 4 } & & FER & Si1 \\
\hline Structure 1 & Si34, Si66, Si82, Si110, Si121, and Si145. & Si4, Si15, Si36, and Si73. & Si2 \\
\hline Structure 2 & Si35, Si67, Si83, Si111, Si122, and Si146. & Si14, Si27, Si43, and Si97. & Si3 \\
\hline Structure 3 & Si1, Si33, Si65, Si81, Si109, and Si124. & Si1, Si47, Si51, and Si106. & Si4 \\
\hline Structure 4 & Si17, Si36, Si52, Si84, Si99, and Si123. & Si25, Si35, Si62, and Si81. & \\
\hline Structure 5 & Si4, Si33, Si81, Si112, Si122, and Si145. & Si43. Si51, Si72, and Si100. & \\
\hline Structure 6 & Si3, Si35, Si65, Si98, Si186, and Si202. & Si16, Si34, Si55, and Si72. & \\
\hline Structure 7 & Si19, Si84, Si121, Si174, Si201, and Si210. & Si14, Si47, Si72, and Si91. & \\
\hline Structure 8 & Si4, Si19, Si52, Si83, Si124, and Si129. & Si34, Si60, Si73, and Si103. & \\
\hline Structure 9 & Si35, Si66, Si97, Si121, Si148, and Si209. & Si3, Si25, Si81, and Si106. & \\
\hline Structure 10 & Si2, Si51, Si97, Si123, Si145, and Si189. & Si13, Si51, Si90, and Si106. & \\
\hline
\end{tabular}


TABLE 5: Structural Information for MOR zeolite considered in this work

\begin{tabular}{|l|l|}
\hline & Al location sites \\
\cline { 2 - 3 } & MOR \\
\hline Structure 1 & Si3, Si17, Si32, Si43, Si79, Si92, Si106, and Si116. \\
\hline Structure 2 & Si3, Si17, Si32, Si43, Si79, Si93, Si106, and Si115. \\
\hline Structure 3 & Si3, Si17, Si32, Si43, Si80, Si92, Si105, and Si116. \\
\hline Structure 4 & Si3, Si17, Si32, Si43, Si89, Si93, Si105, and Si115. \\
\hline Structure 5 & Si3, Si18, Si32, Si42, Si79, Si92, Si106, and Si116. \\
\hline Structure 6 & Si3, Si18, Si32, Si42, Si79, Si93, Si106, and Si115. \\
\hline Structure 7 & Si3, Si18, Si32, Si42, Si80, Si92, Si105, and Si116. \\
\hline Structure 8 & Si3, Si18, Si32, Si42, Si80, Si93, Si105, and Si115. \\
\hline Structure 9 & Si4, Si17, Si31, Si43, Si79, Si92, Si106, and Si116. \\
\hline Structure 10 & Si4, Si17, Si31, Si43, Si79, Si93, Si106, and Si115. \\
\hline Structure 11 & Si4, Si17, Si31, Si43, Si80, Si92, Si105, and Si116. \\
\hline Structure 12 & Si4, Si17, Si31, Si43, Si80, Si93, Si105, and Si115. \\
\hline Structure 13 & Si4, Si18, Si31, Si42, Si79, Si92, Si106, and Si116. \\
\hline Structure 14 & Si4, Si18, Si31, Si42, Si79, Si93, Si106, and Si115. \\
\hline Structure 15 & Si4, Si18, Si31, Si42, Si80, Si92, Si105, and Si116. \\
\hline Structure 16 & Si4, Si18, Si31, Si42, Si80, Si93, Si105, and Si115. \\
\hline
\end{tabular}

Figure 2 shows the calculated Henry coefficients of linear alkanes in H-TON zeolite at $473 \mathrm{~K}, 498 \mathrm{~K}$, and $523 \mathrm{~K}$. The number of protons is kept one per unit cell to allow a direct comparison with experimental data. Since there are four distinct T-sites aluminium can be located and as the positions and stability of protons in the zeolite are strongly related to its $\mathrm{Al}$ distribution ${ }^{3}$, four different structures were considered in this work, which correspond to the four T-sites.

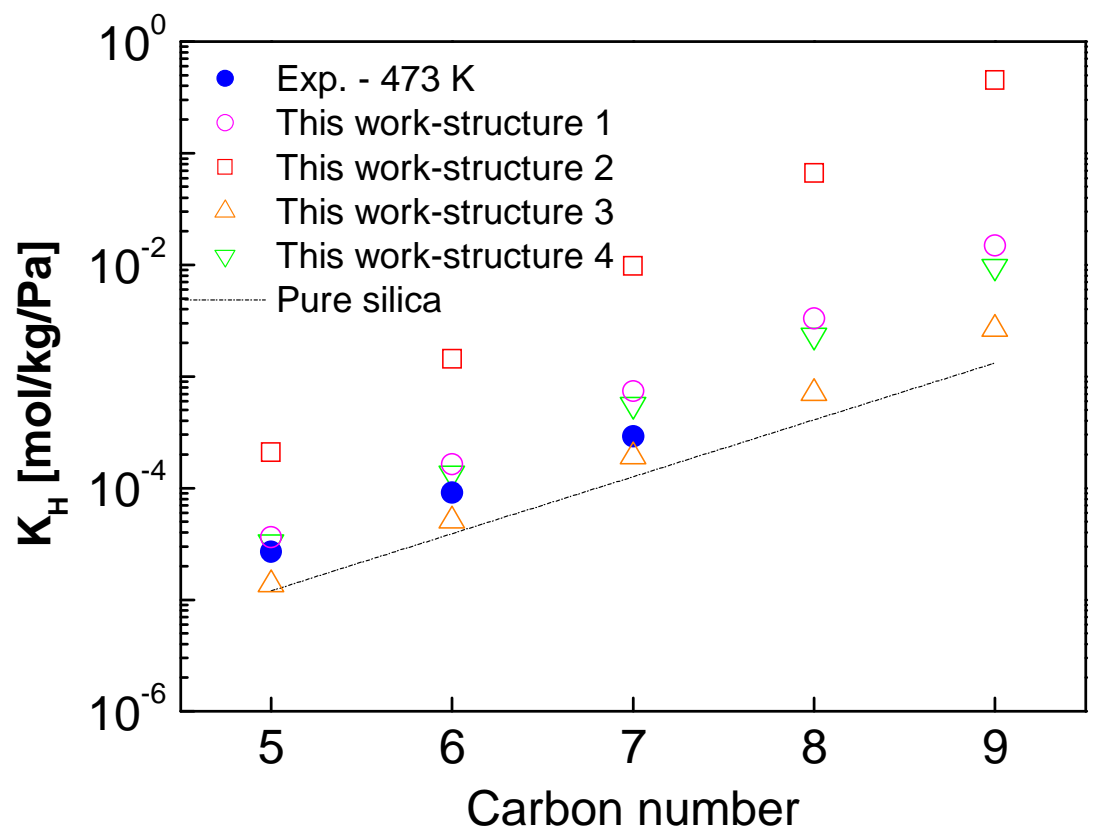



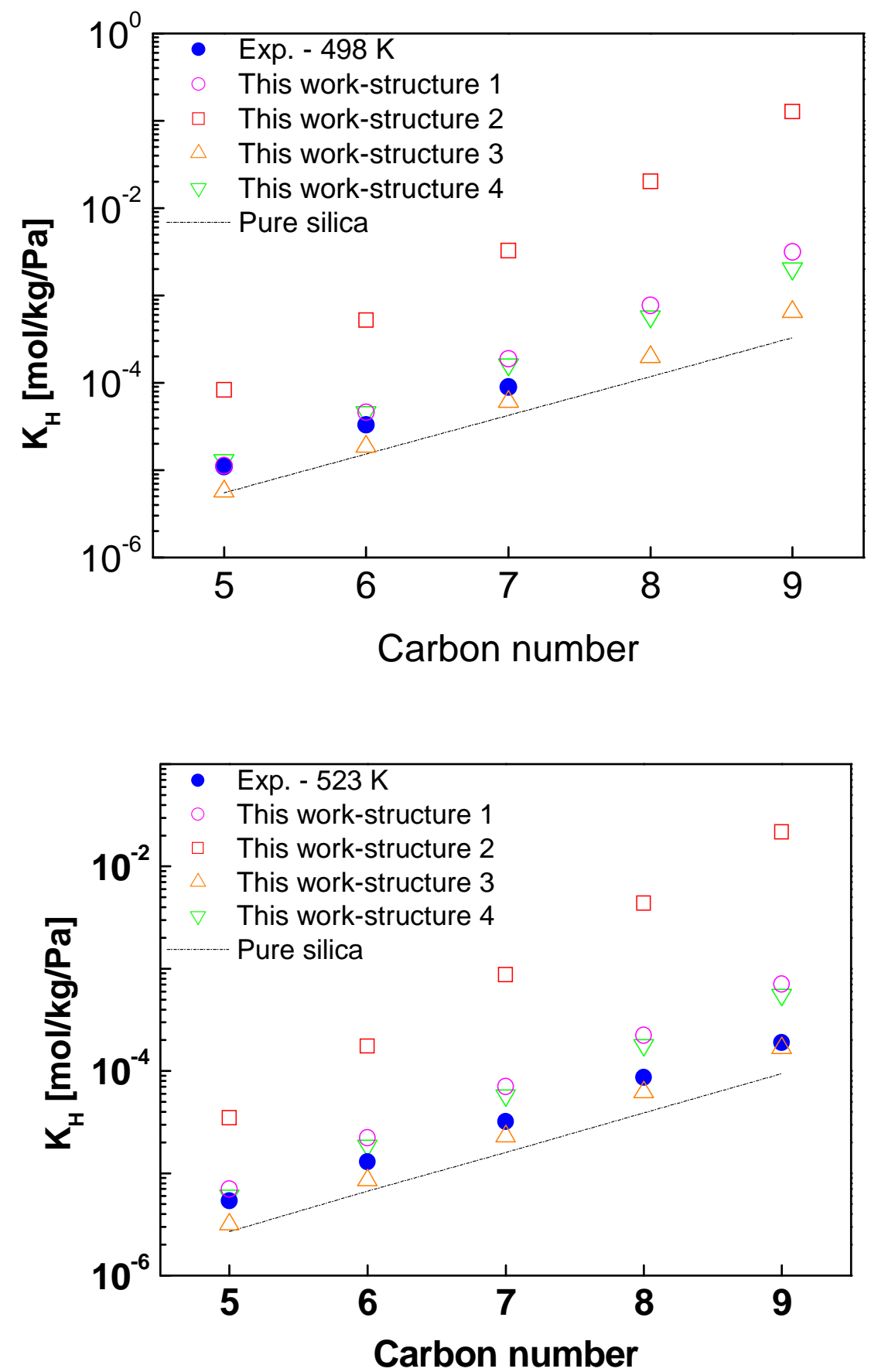

Fig.2 Comparison of the experimental ${ }^{16}$ and simulated Henry coefficients of linear alkanes in H-TON zeolites at $473 \mathrm{~K}, 498 \mathrm{~K}$, and $523 \mathrm{~K}$. Structures 1, 2, 3, and 4 indicate that the $\mathrm{Al}$ atom is located in T-site 1,2, 3, and 4 respectively.

Figure 3 shows the calculated adsorption isotherms of $n$-butane and $n$-hexane in H-FER zeolite at $333 \mathrm{~K}$. The number of protons is kept one per unit cell and four different structures were considered corresponding to the four T-sites. 

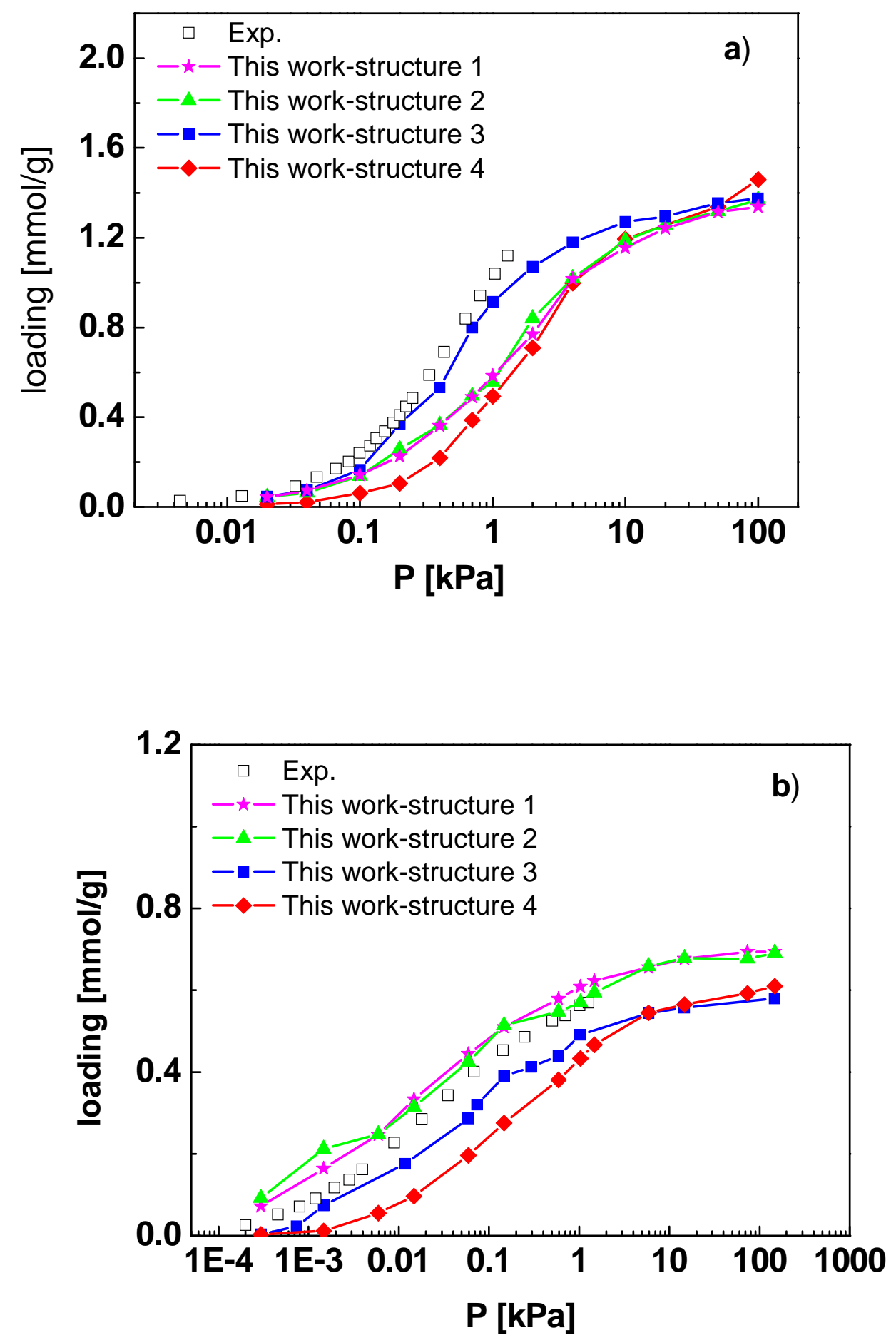

Fig.3 Comparison of the experimental ${ }^{13}$ and simulated adsorption isotherms of a) $n$-butane and b) $n$-hexane in H-FER zeolite at $333 \mathrm{~K}$. Structures 1, 2, 3, and 4 indicate that the $\mathrm{Al}$ atom is located in T-site 1, 2, 3, and 4 respectively. 